\title{
Apetite emocional em situações negativas e padrão alimentar de mulheres adultas
}

\section{Emotional appetite in negative situations and dietary patterns of adult women} Apetito emocional en situaciones negativas y el patrón alimentario de mujeres adultas

\author{
Yanna Letícia Menezes Paiva (iD \\ Escola de Saúde Pública - Fortaleza (CE) - Brasil \\ Leandro Teixeira Cacau (iD \\ Universidade de São Paulo - São Paulo (SP) - Brasil
}

Helena Alves de Carvalho Sampaio (iD

Universidade Estadual do Ceará - Fortaleza (CE) - Brasil

Lia Silveiro Adriano (iD

Universidade de Fortaleza - Fortaleza (CE) - Brasil

Antônio Augusto Ferreira Carioca iD

Universidade de Fortaleza - Fortaleza (CE) - Brasil

Soraia Pinheiro Machado Arruda (iD

Universidade Estadual do Ceará - Fortaleza (CE) - Brasil

Sabrina Dantas Sabry iD

Faculdade Vale do Jaguaribe - Fortaleza (CE) - Brasil

\section{RESUMO}

Objetivo: Verificar se e quais padrões alimentares podem estar associados ao apetite emocional em mulheres acompanhadas pelo Sistema Único de Saúde (SUS). Métodos: Estudo quantitativo, transversal e analítico realizado em 20 Centros de Saúde da Família do SUS, entre 2016 e 2017, com mulheres de 19 a 59 anos, em idade reprodutiva e alfabetizadas. Coletaram-se dados de peso, altura e circunferência da cintura, e aplicou-se o Questionário de Apetite Emocional (QUEAPEM), para avaliar o apetite emocional, e o Questionário de Frequência Alimentar, para avaliar o consumo alimentar, analisado na forma de padrões alimentares derivados por análise fatorial por meio de testes de esfericidade de Barllet e Kayse-Meyer-Olkin. Verificou-se relação entre os padrões alimentares e a dimensão do apetite emocional pelo teste de Spearman, considerando significativos os valores de $p<0,05$. Resultados: Ao total, 149 mulheres participaram do estudo, com idade média de $34,3 \pm 8,6$ anos. A maioria das mulheres $(n=88 ; 59 \%)$ encontra-se com excesso de peso, sendo $25,5 \%(n=38)$ com obesidade. Identificaram-se três padrões alimentares que juntos explicaram $16,8 \%$ da variabilidade da dieta, denominados de "denso em energia", "saudável" e "tradicional". Houve correlação entre o padrão alimentar "tradicional" e a presença de apetite emocional em situações negativas $(p<0,05)$, não havendo correlação com os demais padrões. Conclusão: A identificação de três padrões alimentares permitiu um melhor entendimento da alimentação das mulheres estudadas, além de detectar uma associação inversa, embora fraca, entre o padrão alimentar "tradicional" e o apetite emocional em situações negativas.

Descritores: Apetite; Comportamento Alimentar; Consumo Alimentar.

\section{ABSTRACT}

Objective: To check whether and what dietary patterns may be associated with emotional appetite in women served by the Brazilian Unified Health System (Sistema Único de Saúde - SUS). Methods: This quantitative analytical cross-sectional study was conducted in 20 SUS Family Health Centers between 2016 and 2017 with literate women aged 19 to 59 years at reproductive age. Data on weight, height, and waist circumference were collected and the Emotional Appetite Questionnaire (Questionário de Apetite Emocional - QUEAPEM) was applied to assess emotional appetite. The Food Frequency Questionnaire was used to assess food intake in the form of dietary patterns derived from factor analysis using Barllet's and Kayse-Meyer-Olkin sphericity tests. The relationship between dietary patterns and emotional appetite dimension was checked by the Spearman's test with significant values set at $p<0.05$. Results: Participants were 149 women with a mean age of $34.3 \pm 8.6$ years. Most women ( $n=$

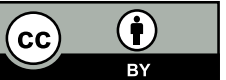


88, 59\%) exhibited excess weight, with $25.5 \%(n=38)$ of them being obese. Three dietary patterns were identified and together explained $16.8 \%$ of the variability of the diet. They were labelled "energy dense", "healthy" and "traditional". There was a correlation between the "traditional" dietary pattern and presence of emotional appetite in negative situations $(p<0.05)$, with no correlation with the other patterns. Conclusion: The identification of three dietary patterns allowed a better understanding of the diet of the studied women and detected an inverse, although weak, association between the "traditional" dietary pattern and emotional appetite in negative situations.

Descriptors: Appetite; Eating Behavior; Food Consumption.

\section{RESUMEN}

Objetivo: Verificar si y cuáles son los patrones alimentarios que se puede asociar con el apetito emocional de mujeres asistidas en el Sistema Único de Salud (SUS). Métodos: Estudio cuantitativo, transversal y analítico realizado en 20 Centros de Salud de la Familia del SUS entre 2016 y 2017 con mujeres entre 19 y 59 años con edad reproductiva y alfabetizadas. Se recogieron los datos del peso, la altura y la circunferencia de la cintura y se ha aplicado el Cuestionario de Apetito Emocional para evaluar el apetito emocional y el Cuestionario de Frecuencia Alimentaria para evaluar el consumo alimentario analizado en forma de patrones alimentarios derivados por el análisis factorial a través de las pruebas de Barllet y de Kayse-Meyer-Olkin. Se ha verificado la relación entre los patrones alimentarios y la dimensión del apetito emocional por la prueba de Spearman considerándose significativos los valores de p<0,05. Resultados: En total, 149 mujeres con edad media de 34,3 \pm 8,6 años participaron del estudio. La mayoría de las mujeres $(n=88 ; 59 \%)$ tiene exceso de peso y el $25,5 \%(n=38)$ son obesas. Se ha identificado tres patrones alimentarios que juntos han explicado el 16,8\% de la variabilidad de la dieta los cuales se les han nombrado de "denso en energía", "saludable" y "tradicional". Hubo correlación entre el patrón alimentario "tradicional" y la presencia del apetito emocional en situaciones negativas $(p<0,05)$ sin correlación con los demás patrones. Conclusión: La identificación de tres patrones alimentarios ha permitido una mejor comprensión de la alimentación de las mujeres estudiadas además de identificar una asociación inversa, aunque débil, entre el patrón alimentario "tradicional" y el apetito emocional en situaciones negativas.

Descriptores: Apetito; Conducta Alimentaria; Consumo Alimentario.

\section{INTRODUÇÃO}

De acordo com o Global Burden of Disease (GBD), relatando dados de 2015, há 603,7 milhões de adultos obesos, representando, aproximadamente, um terço da população mundial(1). No Brasil, de acordo com o Sistema de Vigilância de Fatores de Risco e Proteção para Doenças Crônicas por Inquérito Telefônico (Vigitel), houve um crescimento de $26,3 \%$ do excesso de peso nos últimos dez anos, sendo que, no ano de 2016, mais da metade da população adulta brasileira apresentou excesso de peso(2).

O excesso de peso está relacionado ao aumento do risco de desenvolvimento de uma ampla lista de doenças, com destaque para doenças cardiovasculares, diabetes mellitus tipo 2, hipertensão, dislipidemia e diversos tipos de câncer ${ }^{(3)}$. O seu desenvolvimento é multifatorial e pode ser influenciado por questões individuais ou, ainda, por características sociais e comportamentais, como oscilações de humor, baixa autoestima, ansiedade e depressão, considerando-se também o apetite emocional ${ }^{(4)}$.

O apetite emocional, por sua vez, é definido como uma tendência do aumento do ato de comer em resposta a gatilhos emocionais, ou seja, quando não há uma verdadeira necessidade fisiológica de alimentos ${ }^{(5)}$. Indivíduos com apetite emocional, quando expostos a situações de emoção intensa, respondem com um aumento da ingestão, não havendo redução ou supressão de fome e saciedade, o que é esperado biologicamente ${ }^{(6)}$.

Estudos demonstram que a desregulação emocional se caracteriza como uma incapacidade de reconhecer e controlar respostas emocionais intensas, em que indivíduos com níveis de estresse elevados tornam-se indiferentes aos seus próprios sinais biológicos de fome e saciedade, confundindo excitação emocional e fome, produzindo, desta forma, um mecanismo compensatório(7-9).

A investigação do consumo alimentar é relevante na compreensão da relação entre dieta e obesidade, entretanto a análise tradicional da ingestão alimentar é focada no consumo de nutrientes ou alimentos isolados, gerando uma visão reducionista da dieta. A análise de padrões alimentares permite uma visão holística da dieta, uma vez que as pessoas consomem um conjunto de alimentos variados em refeições complexas. Assim, a análise de padrões alimentares favorece a compreensão dos processos de prevenção e tratamento de doenças e vem se constituindo como ferramenta fundamental para os programas de intervenção nutricional ${ }^{(10)}$.

Tendo em vista que indivíduos que possuem apetite emocional são mais propensos ao desenvolvimento de sobrepeso e obesidade ${ }^{(11,12)}$, e que, pelo levantamento em base de dados, não há estudos na literatura sobre padrões 
alimentares e apetite emocional, faz-se necessário o estudo dessa temática como forma de compreender possíveis fatores associados e buscar novas ferramentas para avaliar associações com apetite emocional. No momento, esse tipo de análise não tem sido destacado nas políticas públicas de saúde no Brasil, apontando que o presente estudo contribuirá para facilitar a adoção de estratégias de intervenção mais embasadas para promoção da saúde. Dessa forma, este estudo tem como objetivo verificar se e quais padrões alimentares podem estar associados ao apetite emocional em mulheres acompanhadas pelo Sistema Único de Saúde (SUS).

\section{MÉTODOS}

Trata-se de um estudo com delineamento transversal, quantitativo e analítico, que utiliza dados de um estudo maior, intitulado "Projeto Prevendo - Saúde, Envelhecimento, Dieta e Inflamação: desenvolvimento, validação e normatização de instrumentos para promoção da saúde e prevenção de doenças crônicas não transmissíveis". 0 Projeto Prevendo visa à normatização do uso de indicadores na rotina de atendimento do SUS, levando a um maior sucesso na promoção da saúde e no combate às doenças crônicas não transmissíveis (DCNT). O estudo citado foi realizado em 20 Unidades de Atenção Primária à Saúde (UAPS) integrantes da Atenção Básica da Secretaria Municipal de Saúde da Prefeitura Municipal de Fortaleza, estado do Ceará, Nordeste do Brasil. Essas UAPS prestam atendimento por meio do SUS na cidade de Fortaleza. Para a seleção das UAPS, foram contempladas todas as regionais da cidade, realizando-se sorteios entre as UAPS das secretarias regionais, assegurando-se, ao menos, duas UAPS de cada uma das seis secretarias de Fortaleza.

A população integrante do estudo Projeto Prevendo foi composta por mulheres usuárias do SUS e cadastradas nas UAPS selecionadas. De acordo com a literatura, as mulheres estão mais propensas a alterações na regulação do controle emocional, que levam a estímulos de compensação alimentar ${ }^{(9)}$, sendo, dessa maneira, incluídas apenas mulheres no estudo. A amostra do Projeto Prevendo foi de conveniência e composta por 450 mulheres acompanhadas nas UAPS, selecionadas aleatoriamente enquanto aguardavam atendimento.

Desse modo, os critérios de inclusão no estudo foram: mulheres adultas ( $\geq 20$ e $<60$ anos), em idade reprodutiva, alfabetizadas e que estivessem sendo atendidas nas respectivas UAPS, sem restrições mentais e físicas que impossibilitassem a compreensão do questionário e da entrevista, bem como que impedissem a obtenção das medidas antropométricas. Como critérios de exclusão: mulheres no período da menopausa e que não possuíam todos os dados necessários para este estudo em questão. Após exclusão, a amostra final incluída neste estudo foi de 149 mulheres.

A coleta de dados aconteceu entre os anos de 2016 a 2017, sendo dividida em duas etapas. Na primeira, coletaram-se dados de identificação (idade), socioeconômicos (escolaridade, renda, raça autodeclaracada e estado civil) e clínicos (hábito de fumar e ingestão de bebidas alcoólicas), além de ter sido aplicado o questionário de apetite emocional (QUEAPEM) $)^{(13)}$, terem sido aferidas as medidas antropométricas de peso, altura, circunferência da cintura (CC), e calculado o índice de massa corporal (IMC) $(\mathrm{kg} / \mathrm{m} 2)$. Realizaram-se as classificações do IMC e CC de acordo com as recomendações da World Health Organization (WHO) ${ }^{(14)}$. A CC das mulheres foi considerada adequada, ou seja, sem risco de complicações, quando menor do que $80 \mathrm{~cm}$. As categorias de obesidade, agrupadas em uma só categoria, resultaram em: IMC menor que $18,50 \mathrm{~kg} / \mathrm{m}^{2}$ - magreza; IMC de $18,50 \mathrm{~kg} / \mathrm{m}^{2}$ a $24,99 \mathrm{~kg} / \mathrm{m}^{2}$ - eutrofia; de $25,0 \mathrm{~kg} / \mathrm{m}^{2}$ a $29,99 \mathrm{~kg} / \mathrm{m}^{2}$ - sobrepeso; $\geq 30 \mathrm{~kg} / \mathrm{m}^{2}$ - obesidade.

O apetite emocional foi avaliado por meio do Questionário de Apetite Emocional (QUEAPEM) $)^{(13)}$, um questionário adaptado para o português brasileiro com base no Emotional Appetite Questionnaire (EMAQ) $)^{(13)}$. O QUEAPEM ${ }^{(13)}$ é constituído de 22 perguntas sobre tendência de comer em resposta a emoções positivas e negativas (14 itens), sendo 9 negativas (triste, entediado, com raiva, ansioso, frustrado, cansado, deprimido, assustado, solitário) e 5 positivas (confiante, feliz, relaxado, brincalhão, entusiasmado), além de situações positivas e negativas (8 itens), sendo 5 negativas (quando está sob pressão, após uma discussão acalorada, após uma tragédia com alguém, após o fim de um relacionamento, após perder dinheiro ou bens) e 3 positivas (quando se apaixona, quando está envolvido em um passatempo agradável, após receber boas notícias). Cada item é classificado numa escala do tipo "Likert" de resposta à quantidade de alimentos ingerida, variando de 1 ponto (muito menos) a 9 pontos (muito mais), sendo que 5 pontos indicam o "mesmo". Para cada item, existe também a opção "não se aplica" ou "não sei". Não há ponto de corte, sendo as emoções e situações negativas e positivas avaliadas como variável contínua.

$\mathrm{Na}$ segunda fase do estudo, coletaram-se os dados referentes ao consumo alimentar, obtidos por meio do Questionário de Frequência Alimentar (QFA) desenvolvido e validado para o Estudo Longitudinal de Saúde do Adulto (ELSA-Brasil)(15), o QFA-Elsa. Esse instrumento possui 114 itens divididos em 7 grupos alimentares: pães, cereais e tubérculos; frutas, frutas secas e em calda; verduras, legumes e leguminosas; ovos, carnes, leites e derivados; 
massas; doces e bebidas. O QFA-Elsa foi aplicado por contato telefônico com mulheres participantes da primeira etapa do estudo. Os alimentos informados transformaram-se em gramas e mililitros ${ }^{(16)}$.

Como meio de minimizar o número de variáveis relativas ao consumo alimentar, dividiram-se os alimentos em grupos de alimentos. Inicialmente, de acordo com a sua composição nutricional, contudo excluíram-se alimentos que apresentaram frequência de ingestão menor do que $5 \%$, sendo eles a maionese light, o acarajé e o vinho. Ao final, determinou-se 27 grupos alimentares (Quadro I).

A identificação dos padrões alimentares ocorreu por meio de análise fatorial, utilizando-se o método de estimação por componentes principais (ACP), sendo os fatores obtidos rotacionados por meio de rotação ortogonal varimax, por ser o método mais utilizado para redução de dados com perda mínima de informações, com combinações lineares entre os grupos alimentares que explicam a maior parte da variação na dieta dos indivíduos ${ }^{(10)}$.

Para testar a aplicabilidade da análise fatorial nos dados de consumo alimentar, realizaram-se os testes de esfericidade de Barllet e Kayse-Meyer-Olkin (KMO). Utilizaram-se como critérios para a retenção dos fatores valores de eigenvalue $>1$, de acordo com o gráfico de Cattel, em que o eigenvalue representa a quantidade da variância que pode ser explicada por um fator. Consideraram-se os grupos alimentares com valor de carga fatorial maior ou igual a +0,3 e menor ou igual a $-0,3$ como componentes importantes para cada padrão alimentar ${ }^{(10)}$. A nomeação dos padrões alimentares ocorreu de acordo com a composição nutricional dos alimentos de cada componente e baseada em nomenclaturas já utilizadas em outros estudos em que a composição dos padrões foram semelhantes.

Para verificar a relação entre os padrões alimentares obtidos com a dimensão do apetite emocional, realizou-se o teste de correlação de Spearman, segundo emoções e situações (positivas e negativas). Utilizando-se o software SPSS, versão 22.0, para as análises, sendo considerados como significativos os valores de $p<0,05$.

O presente estudo seguiu as recomendações da Resolução n. ${ }^{\circ} 466 / 12$ do Conselho Nacional de Saúde e, como citado anteriormente, integra o "Projeto Prevendo - Saúde, Envelhecimento, Dieta e Inflamação: desenvolvimento, validação e normatização de instrumentos para promoção da saúde e prevenção de doenças crônicas não transmissíveis". O projeto em questão obteve a aprovação do Comitê de Ética em Pesquisa com Seres Humanos da Universidade Estadual do Ceará (Parecer $n^{\circ} 314.351$ ). Todas as participantes assinaram um Termo de Consentimento Livre e Esclarecido (TCLE) e foram informadas de forma tácita quanto aos objetivos da pesquisa.

\section{RESULTADOS}

Das 450 mulheres avaliadas no Projeto Prevendo, somente 149 possuíam todos os dados completos de consumo alimentar e de apetite emocional. A média de idade do grupo foi $34,3 \pm 8,6$ anos, sendo que $65,1 \%$ ( $n=97$ ) das mulheres possuíam 30 ou mais anos de idade, $69,1 \%(n=103)$ possuía nove ou mais anos de estudo, $73,2 \%(n=109)$ se autodeclarava parda, $89,9 \%(n=134)$ possuía renda familiar de um a três salários mínimos, 96,6\% $(n=144)$ não eram tabagistas, $91,3 \%(n=136)$ não eram consumidoras de bebidas alcoólicas e $51 \%(n=76)$ eram casadas. Houve predomínio de mulheres com circunferência da cintura elevada $(82,6 \% ; n=123)$ e excesso de peso, sendo $33,5 \%$ $(n=50)$ com sobrepeso e $25,5 \%$ ( $n=38$ ) com obesidade. (Tabela I).

Depois de confirmada a adequação dos dados pelo coeficiente $\operatorname{KMO}(0,645)$ e pelo teste de esfericidade de Bartlett $(p<0,001)$, os 27 grupos alimentares foram incluídos na análise (Quadro I), derivando-se três padrões alimentares, que foram denominados de "denso em energia", "saudável" e "tradicional", que juntos explicaram 33,8\% da variabilidade total da dieta das mulheres avaliadas (Tabela II).

O padrão "denso em energia" foi o que mais representou o consumo alimentar dessas mulheres, explicando $16,4 \%$ da variabilidade total da dieta, sendo composto por produtos de panificação, como bolos e biscoitos, massas, tubérculos, ovos, laticínios desnatados, gorduras, carnes, embutidos, lanches, alimentos regionais, como cuscuz, farinha de mandioca, pirão e vatapá, doces, refrigerantes, bebidas alcoólicas e feijoada. No padrão "saudável" estavam inseridos os cereais, frutas, legumes e verduras, oleaginosas, aves, pescados, sopas e sushi. Esse padrão explicou 9,3\% da variabilidade total da dieta. Os alimentos retidos no padrão "tradicional" foram arroz, pães, biscoitos e bolos, leguminosas, laticínios integrais e sucos, com cargas negativas para cereais integrais, laticínios desnatados e bebidas alcoólicas. Este explicou 8,0\% da variabilidade total da dieta (Tabela II).

Os resultados referentes ao questionário sobre apetite emocional foram obtidos pela média de variáveis contínuas, dividido em emoções e situações negativas para a presença de apetite emocional e em emoções e situações positivas para a ausência de apetite emocional. Houve uma maior prevalência de respostas para emoções e situações positivas (Tabela III). 
Quando analisada a relação entre o apetite emocional e os padrões alimentares, observou-se correlação inversa do apetite emocional com situações negativas e padrão alimentar "tradicional" $(r=-0,18 ; p<0,05)$. No entanto, para os padrões "denso em energia" e "saudável", não foram encontradas significâncias estatísticas na associação com a escala de apetite emocional (Tabela IV).

Quadro I - Agrupamento dos alimentos relatados pelas mulheres participantes do estudo. Fortaleza, Brasil, 2020.

\begin{tabular}{|c|c|}
\hline Grupos de alimentos & Descrição \\
\hline Arroz & Arroz branco \\
\hline Cereais & Arroz integral, aveia, pão integral, pão light \\
\hline Pão & Pão francês, pão doce \\
\hline Biscoitos e Bolos & Bolo, biscoito salgado, biscoito doce, biscoito doce com recheio \\
\hline Massas & Macarrão, macarrão instantâneo \\
\hline Tubérculos & Purê, batata doce \\
\hline Frutas, Verduras e Legumes (FVL) & Todas as frutas, verduras e legumes \\
\hline Leguminosas & Feijão, grão de bico, vagem \\
\hline Oleaginosa & Castanha de caju \\
\hline Ovo & Ovo cozido, ovo frito \\
\hline Laticínios Desnatados & Leite desnatado, queijo minas frescal, requeijão light, iogurte light \\
\hline Laticínios Integrais & Leite integral, leite semidesnatado, queijo muçarela, requeijão, iogurte integral \\
\hline Gorduras & Margarina, maionese \\
\hline Carnes & Carne com osso, bife, suíno, estrogonofe, fígado, dobradinha \\
\hline Aves & Peito de frango, frango frito, frango cozido \\
\hline Embutidos & Linguiça, hambúrguer, peito de peru, presunto, bacon \\
\hline Pescados & Peixe cozido, peixe frito, sardinha, camarão, caranguejo \\
\hline Lanches & Empada, pastel, cachorro quente, pipoca, batata frita, pão de queijo, pizza \\
\hline Sopa & Sopa de legumes, sopa instantânea \\
\hline Regional & Vatapá, pirão, cuscuz, farinha de mandioca \\
\hline Doces & $\begin{array}{l}\text { Sorvete, picolé, caramelo, gelatina, achocolatado, chocolate, pudim, doce de fruta, mel, } \\
\text { barra de cereal, açúcar, torta doce, salada de fruta completa }\end{array}$ \\
\hline Refrigerante & Refrigerante normal, Refrigerante light \\
\hline Chá e Café & Café, chá \\
\hline Sucos & Suco natural, suco industrializado, suco artificial \\
\hline Bebidas Alcoólicas & Cerveja, vinho tinto, cachaça \\
\hline Sushi & Sushi \\
\hline Feijoada & Feijoada \\
\hline
\end{tabular}


Tabela I - Distribuição das mulheres usuárias do Sistema Único Brasileiro avaliadas $(n=149)$, segundo as características socioeconômicas e antropométricas. Fortaleza, Brasil, 2020.

\begin{tabular}{|c|c|c|}
\hline Variáveis & $n$ & $\%$ \\
\hline \multicolumn{3}{|l|}{ Idade } \\
\hline$<30$ anos & 52 & 34,9 \\
\hline$\geq 30$ anos & 97 & 65,1 \\
\hline \multicolumn{3}{|l|}{ Anos de estudo } \\
\hline$<9$ anos & 46 & 30,9 \\
\hline$\geq 9$ anos & 103 & 69,1 \\
\hline \multicolumn{3}{|l|}{ Raça autodeclarada } \\
\hline Branca & 31 & 20,8 \\
\hline Negra & 9 & 6,0 \\
\hline Parda & 109 & 73,2 \\
\hline \multicolumn{3}{|l|}{ Estado civil } \\
\hline Solteira & 68 & 45,6 \\
\hline Casada & 76 & 51,0 \\
\hline Divorciadas & 5 & 3,4 \\
\hline \multicolumn{3}{|l|}{ Renda familiar } \\
\hline < 1 salário mínimo & 15 & 10,1 \\
\hline 1 a 3 salários mínimos & 134 & 89,9 \\
\hline \multicolumn{3}{|l|}{ Tabagismo } \\
\hline Não & 144 & 96,6 \\
\hline Sim & 5 & 3,4 \\
\hline \multicolumn{3}{|l|}{ Consumo de álcool } \\
\hline Não & 136 & 91,3 \\
\hline Sim & 13 & 8,7 \\
\hline \multicolumn{3}{|l|}{ Circunferência da cintura } \\
\hline Adequada & 26 & 17,4 \\
\hline Elevada & 123 & 82,6 \\
\hline \multicolumn{3}{|l|}{ Estado nutricional } \\
\hline Baixo peso & 4 & 2,7 \\
\hline Eutrofia & 57 & 38,3 \\
\hline Sobrepeso & 50 & 33,5 \\
\hline Obesidade & 38 & 25,5 \\
\hline
\end{tabular}

Tabela II - Distribuição das cargas fatoriais dos padrões alimentares identificados. Fortaleza, Brasil, 2020.

\begin{tabular}{|c|c|c|c|}
\hline \multirow[b]{2}{*}{ Grupo alimentar } & \multicolumn{3}{|c|}{ Padrão alimentar } \\
\hline & Denso em energia & Saudável & Tradicional \\
\hline Lanches & 0,657 & $-0,036$ & 0,143 \\
\hline Feijoada & 0,634 & 0,123 & $-0,103$ \\
\hline Doces & 0,624 & 0,205 & 0,260 \\
\hline Tubérculos & 0,563 & 0,153 & $-0,035$ \\
\hline Gordura & 0,559 & $-0,114$ & 0,103 \\
\hline Massas & 0,537 & 0,053 & 0,236 \\
\hline Ovo & 0,519 & 0,290 & $-0,074$ \\
\hline Refrigerante & 0,507 & $-0,269$ & 0,011 \\
\hline Biscoitos e bolos & 0,475 & 0,065 & 0,550 \\
\hline Carnes & 0,448 & $-0,067$ & $-0,128$ \\
\hline Regional & 0,448 & 0,262 & 0,215 \\
\hline Embutidos & 0,393 & 0,052 & 0,108 \\
\hline Laticínios desnatados & 0,310 & 0,327 & $-0,422$ \\
\hline Bebidas alcoólicas & 0,300 & $-0,066$ & $-0,310$ \\
\hline Pescados & 0,185 & 0,735 & 0,029 \\
\hline FVL & $-0,009$ & 0,651 & 0,232 \\
\hline Sushi & $-0,196$ & 0,635 & 0,185 \\
\hline Sopa & $-0,020$ & 0,433 & $-0,012$ \\
\hline Aves & 0,106 & 0,429 & 0,063 \\
\hline Oleaginosa & 0,060 & 0,408 & $-0,270$ \\
\hline Cereal integral & 0,093 & 0,404 & $-0,311$ \\
\hline Pão & 0,091 & 0,136 & 0,688 \\
\hline Arroz & 0,042 & $-0,296$ & 0,663 \\
\hline Laticínios integrais & $-0,002$ & 0,024 & 0,506 \\
\hline Leguminosas & 0,272 & 0,138 & 0,336 \\
\hline Sucos & 0,209 & 0,071 & 0,318 \\
\hline
\end{tabular}

Em destaque, carga fatorial de importância maiores que 0,3 (indicando correlação com o padrão) e menores que -0.3 (indicando correlação inversa com o padrão) 
Tabela III - Presença de apetite emocional nas mulheres avaliadas. Fortaleza, Brasil, 2020.

\begin{tabular}{lcc}
\hline Apetite emocional & Média & DP \\
\hline Emoções negativas & 3,84 & 1,15 \\
Emoções positivas & 5,32 & 1,08 \\
Situações negativas & 2,87 & 1,27 \\
Situações positivas & 5,04 & 1,18 \\
\hline
\end{tabular}

DP: desvio-padrão

Tabela IV - Correlação entre apetite emocional e padrões alimentares encontrados nas mulheres avaliadas. Fortaleza, Brasil, 2020.

\begin{tabular}{|c|c|c|c|c|c|c|}
\hline \multirow[t]{2}{*}{ Apetite emocional } & \multicolumn{2}{|c|}{ Denso em energia } & \multicolumn{2}{|c|}{ Saudável } & \multicolumn{2}{|c|}{ Tradicional } \\
\hline & $\mathbf{r}$ & $p^{*}$ & $\mathbf{r}$ & $\mathbf{p}^{*}$ & $\mathbf{r}$ & $\mathbf{p}^{*}$ \\
\hline Emoções negativas & 0,055 & 0,502 & $-0,005$ & 0,948 & $-0,071$ & 0,387 \\
\hline Emoções positivas & 0,043 & 0,601 & $-0,102$ & 0,214 & 0,038 & 0,645 \\
\hline Situações negativas & $-0,045$ & 0,585 & 0,062 & 0,453 & $-0,185$ & 0,024 \\
\hline Situações positivas & 0,029 & 0,724 & 0,039 & 0,636 & 0,049 & 0,549 \\
\hline
\end{tabular}

${ }^{*}$ Correlação de Spearman

\section{DISCUSSÃO}

Após busca nas bases de dados, observou-se que este é o primeiro estudo a avaliar a relação entre o apetite emocional e os padrões alimentares derivados por meio de análise fatorial. No presente estudo, foram identificados três padrões alimentares, que foram capazes de explicar $33,8 \%$ da variabilidade total da dieta das mulheres avaliadas. O padrão alimentar "denso em energia", composto prioritariamente por alimentos de alta densidade energética e alto grau de processamento, como produtos de panificação, lanches fast-foods, refrigerantes, bebidas alcoólicas e feijoada, similar ao encontrado na literatura ${ }^{(17)}$. O padrão "saudável", composto por cereais, frutas, legumes e verduras, oleaginosas, aves, pescados, sopas e sushi, um padrão semelhante ao encontrado em outros estudos que também o denominaram de "saudável"(17,18). Por último, o padrão alimentar "tradicional", composto por alimentos bastante consumidos pela população brasileira, como arroz, feijão, pães e sucos ${ }^{(17,19)}$.

Em relação ao apetite emocional, somente o padrão alimentar "tradicional" apresentou correlação significativa. Observou-se uma relação inversa entre esse padrão e o apetite emocional em situações negativas $(r-0,18 ; p<0,05)$, ou seja, as mulheres que aumentam o consumo alimentar em situações negativas tendem a ter menor aderência ao padrão alimentar tradicional. Como descrito na literatura, esse padrão é aquele que apresenta alimentos e preparações mais prevalentes da dieta do país ou local do estudo ${ }^{(20)}$. No Brasil, o padrão tradicional é comumente descrito como arroz e feijão, laticínios integrais, carboidratos refinados e bebidas à base de frutas ${ }^{(17,19)}$. Embora seja pobre em frutas, verduras, legumes, fibras e com alto consumo de gorduras saturadas e colesterol, ainda assim pode ser considerado mais saudável do que o padrão denso em energia, composto prioritariamente por alimentos ultraprocesssados, que são ricos em açúcares e gorduras ${ }^{(21)}$.

Desse modo, isso explicaria, em partes, o motivo de mulheres em situações negativas terem uma baixa adesão ao padrão tradicional, uma vez que, de acordo com a literatura, indivíduos em situações negativas tendem a ter um aumento no consumo de lanches, alimentos gordurosos, doces e alimentos calóricos ${ }^{(22-25)}$, características inerentes aos ultraprocessados, que estiveram presentes no padrão "denso em energia". A regulação emocional e de humor estão associadas com o consumo de alimentos hedônicos, que são aqueles que levam à tendência do comer pelo sabor agradável e prazeroso, como chocolates e doces ${ }^{(26)}$, presentes no padrão alimentar "denso em energia". Contudo essa hipótese não atende ao padrão alimentar "saudável", que, assim como o "denso em energia", não foi significativo do ponto de vista estatístico.

Vale destacar que estudos anteriores demonstraram ligações entre o apetite emocional e sintomas aumentados de depressão(27), ansiedade ${ }^{(28)}$ e comportamentos alimentares desordenados ${ }^{(29)}$. Um estudo com mulheres não obesas e que não estavam seguindo dietas para perda de peso, que utilizou o Dutch Eating Behavior Questionnaire e buscou investigar o efeito do estado negativo afetivo no consumo alimentar, verificou que o consumo aumentou significativamente durante uma situação negativa. Além disso, esses autores observaram que o consumo alimentar em situações negativas é motivado por recompensas e que indivíduos com dietas restritas possuem maior risco de consumo excessivo durante uma situação negativa ${ }^{(30)}$. Em outro estudo, com universitários, no qual os autores 
também utilizaram o Dutch Eating Behavior Questionnaire( ${ }^{(31)}$, o apetite emocional em emoções negativas, como propensão ao tédio e dificuldades na regulação emocional, previram, independentemente, a probabilidade do aumento do consumo alimentar em resposta a essas emoções negativas, embora os autores não tenham estudado a composição desse consumo alimentar.

O presente estudo demonstrou que as mulheres em situações negativas tendem a ter menor aderência o padrão "tradicional", composto por arroz, feijão, pães e sucos de frutas. O aumento do consumo alimentar é potencializado em situações e emoções negativas, e geralmente é constituído de alimentos ultraprocessados, isto é, ricos em açúcar, gorduras e alta densidade energética ${ }^{(22-25)}$. Dessa forma, este estudo traz um resultado interessante, auxiliando na hipótese sobre o aumento do consumo de alimentos ultraprocessados e, consequentemente, diminuição do consumo de alimentos tradicionais e saudáveis em situações e emoções negativas, mesmo que não tenham sido encontradas diferenças estatísticas para os padrões "denso em energia" e "saudável".

Além disso, vale destacar a relevância deste estudo na atual situação da pandemia do novo coronavírus (COVID-19), pois, frente ao distanciamento social instituído em diversos países, a população precisou ficar confinada e, com isso, passou a realizar mais refeições em casa. Tanto a pandemia da COVID-19 quanto a quarentena, tornaram-se motivo de estresse, ansiedade, depressão e sofrimento psicossocial, tendo impactos diretos na alimentação( ${ }^{(32)}$. Como visto anteriormente, emoções e situações negativas podem influenciar no consumo alimentar dos indivíduos, refletindo em um aumento do consumo de alimentos ultraprocessados. Assim, estudos estão acontecendo para tentar mensurar os impactos da quarentena no consumo alimentar da população.

Estudo brasileiro que avaliou o consumo alimentar antes e durante a quarentena identificou que houve um aumento, embora singelo, no consumo de alimentos saudáveis, como frutas, verduras e legumes, e uma estabilização do consumo de ultraprocessados, indicando que não houve o aumento do consumo de ultraprocessados durante a quarentena ${ }^{(33)}$. Entretanto um estudo realizado na Itália identificou que houve aumento no consumo de alimentos denominados de "comfort foods", como chocolates, sobremesas e sorvetes, e diminuição no consumo de frutas e verduras durante a quarentena ${ }^{(34)}$, o que pode estar associado com a diminuição de consumo de alimentos saudáveis ou tradicionais em decorrência de emoções e situações negativas, como as vivenciadas durante o período de isolamento social.

Tais achados devem ser vistos com algumas limitações. Por tratar-se de um estudo transversal, não é possível inferir causalidade. Além disso, a avaliação do consumo alimentar está associada a erros de medida, apesar da identificação de padrões alimentares por técnica estatística consolidada na literatura. Ainda assim, este estudo traz substanciais resultados para a promoção da saúde: 1) a identificação dos três padrões alimentares permitiu avaliar a qualidade da dieta das mulheres avaliadas e, como resultado, observou-se que o padrão "denso em energia" foi o que mais contribuiu para o consumo alimentar, evidenciando uma dieta de baixa qualidade e 2) a menor aderência ao padrão "tradicional" pode indicar que as mulheres consomem menos os alimentos componentes desse padrão em detrimento de outros alimentos, possivelmente ultraprocessados. Suscita, dessa forma, que mais estudos devem ser conduzidos, em especial estudos com amostras maiores, pois tais estudos permitirão melhor direcionamento na promoção à saúde dessa população.

\section{CONCLUSÃO}

Conclui-se que este estudo surge como potencial multiplicador na investigação do apetite emocional, valendose da análise de padrões alimentares. Como contribuição, tem-se a identificação de três padrões alimentares que caracterizaram o consumo alimentar das mulheres avaliadas, além de encontrar uma associação inversa, embora fraca, entre o padrão alimentar "tradicional" e o apetite emocional em situações negativas.

\section{CONFLITOS DE INTERESSE}

Os autores declaram não haver conflitos de interesses.

\section{CONTRIBUIÇÕES}

Yanna Letícia Menezes Paiva e Leandro Teixeira Cacau contribuíram com a aquisição, análise e interpretação dos dados, e a redação do manuscrito. Antônio Augusto Ferreira Carioca, Lia Silveira Adriano e Soraia Pinheiro Machado Arruda contribuíram com a análise e interpretação dos dados. Helena Alves de Carvalho Sampaio e Sabrina Dantas Sabry contribuíram com a elaboração e delineamento do estudo; e a análise e interpretação de dados. Todos os autores revisaram e aprovaram a versão final do artigo. 


\section{REFERENNCIAS}

1. Lin X, Xu Y, Xu J, Pan X, Song X, Shan L, et al. Global Burden of Disease. Global burden of noncommunicable disease attributable to high body mass index in 195 countries and territories, 1990-2017. Endocrine. 2020;69:310-20.

2. Ministério da Saúde (BR). Vigitel Brasil 2019: Vigilancia de Fatores de Risco e Proteção para Doenças Crônicas por Inquérito Telefônico. Brasília: Ministério da Saúde; 2020.

3. Singh GM, Danaei G, Farzadfar F, Stevens GA, Woodward M, Wormser D, et al. The age-specific quantitative effects of metabolic risk factors on cardiovascular diseases and diabetes: a pooled analysis. PLoS One. 2013;8(7):65174.

4. Pinheiro MM, Oliveira JS, Leal VS, Lira PIC, Souza NP, Campos FACS. Prevalence of overweight and associated factors in women in reproductive age in Northeast Brazil. Rev Nutr. 2016;29(5):679-89.

5. Arnow B, Kenardy J, Agras WS. The emotional eating scale: The development of a measure to assess coping with negative affect by eating. Int J Eat Disord. 1995;18(1):79-90.

6. Cebolla A, Barrada JR, van Strien T, Oliver E, Baños R. Validation of the Dutch Eating Behavior Questionnaire (DEBQ) in a sample of Spanish women. Appetite. 2014;73:58-64

7. Brooks SJ, Cedernaes J, Schiöth HB. Increased Prefrontal and Parahippocampal Activation with Reduced Dorsolateral Prefrontal and Insular Cortex Activation to Food Images in Obesity: a Meta-Analysis of fMRI Studies. PLoS One. 2013;8(4).

8. Tan $\mathrm{CC}$, Chow CM. Stress and emotional eating: the mediating role of eating dysregulation. Pers Individ Dif. 2014;66:1-4.

9. Jarcho JM, Tanofsky-Kraff M, Nelson EE, Engel SG, Vannucci A, Field SE, et al. Neural activation during anticipated peer evaluation and laboratory meal intake in overweight girls with and without loss of control eating. Neuroimage. 2015;108:343-53.

10. Santos RDO, Gorgulho BM, Castro MA, Fisberg RM, Marchioni DM, Baltar VT. Principal Component Analysis and Factor Analysis: differences and similarities in Nutritional Epidemiology application. Rev Bras Epidemiol. 2019;22:190041.

11. Péneau S, Ménard E, Méjean C, Bellisle F, Hercberg S. Sex and dieting modify the association between emotional eating and weight status. Am J Clin Nutr. 2013;97(6):1307-13.

12. Konttinen H, Silventoinen K, Sarlio-Lähteenkorva S, Männistö S, Haukkala A. Emotional eating and physical activity self-efficacy as pathways in the association between depressive symptoms and adiposity indicators. Am J Clin Nutr. 2010;92(5):1031-9.

13. Sabry SD, Carioca AAF, Machado S, Adriano L, Sampaio HAC. Adaptação transcultural do Emotional Appetite Questionnaire em mulheres de língua portuguesa. Ciênc Saúde Colet [Internet]. 2018 [acesso em 2020 Out 5]. Disponível em: http://www.cienciaesaudecoletiva.com.br/artigos/adaptacao-transcultural-do-emotionalappetite-questionnaire-em-mulheres-de-lingua-portuguesa/16975? $\mathrm{id}=16975$

14. Word Health Organization. Obesity: preventing and managing the global epidemic: report of a WHO consultation. WHO: Geneva; 2000.

15. Molina MCB, Benseñor IM, Cardoso LO, Velasquez-Melendez G, Drehmer M, Pereira TSS, et al. Reprodutibilidade e validade relativa do Questionário de Frequência Alimentar do ELSA-Brasil. Cad Saude Publica. 2013;29(2):379-89.

16. Pinheiro ABV, Lacerda EMA, Benzecry EH, Gomes MCS, Costa VM. Tabela para Avaliação de Consumo Alimentar em Medidas Caseiras. Rio de Janeiro: Atheneu; 2005.

17. Adriano LS, Sampaio HAC, Arruda SPM, Portela CLM, Melo MLP, Carioca AAF, et al. Healthy dietary pattern is inversely associated with non-alcoholic fatty liver disease in elderly. $\mathrm{Br} \mathrm{J}$ Nutr. 2016;115(12):2189-95.

18. Ternus DL, Henn RL, Bairros F, Costa JS, Olinto MTA. Padrões alimentares e sua associação com fatores sociodemográficos e comportamentais: pesquisa saúde da mulher 2015, São Leopoldo (RS). Rev Bras Epidemiol. 2019;22:190026. 
19. Cattafesta M, Zandonade E, Bissoli NS, Salaroli LB. Dietary patterns of bank employees and their association with socioeconomic, behavioral and labor factors. Ciênc Saúde Colet. 2019;24(10):3909-22.

20. Borges CA, Rinaldi AE, Conde WL, Mainardi GM, Behar D, Slater B. Padrões alimentares estimados por técnicas multivariadas: uma revisão da literatura sobre os procedimentos adotados nas etapas analíticas. Rev Bras Epidemiol. 2015;18(4):837-57.

21. Jessri M, Wolfinger RD, Lou WY, L'Abbé MR. Identification of dietary patterns associated with obesity in a nationally representative survey of Canadian adults: application of a priori, hybrid, and simplified dietary pattern techniques. Am J Clin Nutr. 2017;105(3):669-84.

22. Bell ME, Bhatnagar S, Liang J, Soriano L, Nagy TR, Dallman MF. Voluntary Sucrose Ingestion, Like Corticosterone Replacement, Prevents the Metabolic Deficits of Adrenalectomy. J Neuroendocrinol. 2001;12(5):461-70.

23. Oliver G, Wardle J, Gibson EL. Stress and food choice: a laboratory study. Psychosom Med. 2000;62(6):85365.

24. Elfhag K, Tholin S, Rasmussen F. Consumption of fruit, vegetables, sweets and soft drinks are associated with psychological dimensions of eating behaviour in parents and their 12-year-old children. Public Health Nutr. 2008;11(9):914-23.

25. Konttinen H, Männistö S, Sarlio-Lähteenkorva S, Silventoinen K, Haukkala A. Emotional eating, depressive symptoms and self-reported food consumption. A population-based study. Appetite. 2010;54(3):473-9.

26. Leehr EJ, Krohmer K, Schag K, Dresler T, Zipfel S, Giel KE. Emotion regulation model in binge eating disorder and obesity - a systematic review. Neurosci Biobehav Rev. 2015;49:125-34.

27. Masheb RM, Grilo CM. Emotional overeating and its associations with eating disorder psychopathology among overweight patients with binge eating disorder. Int J Eat Disord. 2006;39(2):141-6.

28. Elfhag K, Morey LC. Personality traits and eating behavior in the obese: Poor self-control in emotional and external eating but personality assets in restrained eating. Eat Behav. 2008;9(3):285-93.

29. Ricca V, Castellini G, Fioravanti G, Lo Sauro C, Rotella F, Ravaldi C, et al. Emotional eating in anorexia nervosa and bulimia nervosa. Compr Psychiatry. 2012;53(3):245-51.

30. Fay SH, Finlayson G. Negative affect-induced food intake in non-dieting women is reward driven and associated with restrained-disinhibited eating subtype. Appetite. 2011;56(3):682-8.

31. Crockett AC, Myhre SK, Rokke PD. Boredom proneness and emotion regulation predict emotional eating. J Health Psychol. 2015;20(5):670-80.

32. Haddad C, Zakhour M, Bou kheir M, Haddad R, Hachach M, Sacre H, et al. Association between eating behavior and quarantine/confinement stressors during the coronavirus disease 2019 outbreak. J Eat Disord. 2020;8:40.

33. Steele EM, Rauber F, Costa CDS, Leite MA, Gabe KT, Louzada MLC, et al. Dietary changes in the NutriNet Brasil cohort during the covid-19 pandemic. Rev Saude Publica. 2020;54:91.

34. Scarmozzino F, Visioli F. Covid-19 and the subsequent lockdown modified dietary habits of almost half the population in an Italian sample. Foods. 2020;9(5):675. 
Endereço do primeiro autor:

Yanna Letícia Menezes Paiva

Escola de Saúde Pública do Ceará

Av. Antônio Justa, 316

Bairro: Meireles

CEP: 60165-090 - Fortaleza - CE - Brasil

E-mail: yanna_m.paiva@hotmail.com

\section{Endereço para correspondência:}

Leandro Teixeira Cacau

Universidade de São Paulo - Faculdade de Saúde Pública

Av. Dr. Arnaldo, 715

Bairro: Cerqueira César

CEP: 01246-904 - São Paulo - SP - Brasil

E-mail: Icacau@usp.br

Como citar: Paiva YLM, Cacau LT, Sampaio HAC, Adriano LS, Carioca AAF, Arruda SPM, et al. Apetite emocional em situações negativas e padrão alimentar de mulheres adultas. Rev Bras Promoç Saúde. 2021;34:10829. 